Research Paper

\title{
Evaluation of anti-infective-related Clostridium difficile-associated colitis using the Japanese Adverse Drug Event Report database
}

Satoshi Nakao ${ }^{1}$, Shiori Hasegawa1 ${ }^{1,2}$, Kazuyo Shimada ${ }^{1}$, Ririka Mukai ${ }^{1}$, Mizuki Tanaka ${ }^{1}$, Kiyoka Matsumoto ${ }^{1}$, Hiroaki Uranishi ${ }^{4}$, Mayuko Masuta ${ }^{5}$, Hiroaki Ikesue ${ }^{2}$, Tohru Hashida ${ }^{2}, K^{2}$ azuhiro Iguchi ${ }^{3}$ and Mitsuhiro Nakamura ${ }^{1 凶}$

1. Laboratory of Drug Informatics, Gifu Pharmaceutical University; Gifu, Japan.

2. Department of pharmacy, Kobe City Medical Center General Hospital; Kobe, Japan.

3. Laboratory of Community Pharmacy, Gifu Pharmaceutical University; Gifu, Japan.

4. Current address: Division of Pharmacy, Nara Medical University Hospital, 840, Shijocho, Kashihara-shi, Nara, 634-8522, Japan.

5. Current address: Division of Pharmacy, Kyoto City Hospital, 1-2, Mibu Higashitakadacho, Nakagyo-ku Kyoto-shi, Kyoto, 604-8845, Japan.

$\triangle$ Corresponding author: Mitsuhiro Nakamura, Ph.D. Professor, Laboratory of Drug Informatics, Gifu Pharmaceutical University, 1-25-4, Daigaku-Nishi, Gifu, 501-1196, JAPAN, Tel: +81-58-230-8100, Fax: +81-58-230-8105, E-mail: mnakamura@gifu-pu.ac.jp.

() The author(s). This is an open access article distributed under the terms of the Creative Commons Attribution License (https://creativecommons.org/licenses/by/4.0/). See http://ivyspring.com/terms for full terms and conditions.

Received: 2020.01.09; Accepted: 2020.03.06; Published: 2020.03.26

\begin{abstract}
Clostridium difficile-associated colitis (CDAC) may cause gastrointestinal illness, ranging in severity from mild diarrhea to fulminant colitis and even mortality. The purpose of this study was to evaluate anti-infective-related CDAC profiles using the Japanese Adverse Drug Event Report (JADER) database.

Methods: We selected case reports of adverse events of CDAC as specified in the Medical Dictionary for Regulatory Activities. The association between the number of administered anti-infectives and aging was evaluated using reporting odds ratio (ROR) and adjusted for covariates using multiple-logistic regression. We also evaluated anti-infective-related CDAC-onset profiles using Weibull shape parameter.

Results: The JADER database contained 534688 reports from April 2004 to June 2018. There were 1222 anti-infective related CDAC events. The top five anti-infectives were as follows: third-generation cephalosporins (Anatomical Therapeutic Chemical (ATC) code: J01DD, 313 cases), fluoroquinolones (ATC code: J01MA, 201 cases), macrolides (ATC code: J01FA, 146 cases), carbapenems (ATC code: J01DH, 143 cases), and penicillins with extended spectrum (ATC code: J01CA, 103 cases). The adjusted RORs ( $95 \%$ confidence interval) in individuals using 1,2 , and $\geq 3$ anti-infectives were 8.88 (7.05-11.18), 9.77 (6.89-13.86), and 18.39 (11.85-28.54), respectively. Moreover, $47.2 \%$ of CDACs occurred within 7 days of anti-infective therapy initiation. The adjusted ROR of interaction terms of $\geq 70$ years $\times 1$ drug was 21.81 (14.56-32.68).

Conclusion: Our results suggest that the number of administered anti-infectives and patient age are associated with CDAC. These data may be particularly beneficial to prescribers and would contribute to improving the management of CDAC.
\end{abstract}

Key words: Clostridium difficile-associated colitis, anti-infective, adverse event, JADER

\section{Introduction}

Clostridium difficile is a ubiquitous anaerobic bacterium that might cause nosocomial diarrhea. Pathogenic $C$. difficile strains produce two major exotoxins, namely, toxin A and toxin B [1-3]. Both toxins stimulate the production of proinflammatory cytokines and exhibit cytotoxic activity. C. difficile infection (CDI) presents in a variety of gastrointestinal manifestations, ranging in severity from asymptomatic carrier status to moderate diarrhea and life-threatening pseudomembranous colitis. [1,2,4-7] 
C. difficile is the causative pathogen in $20 \%-30 \%$ of antibiotic-associated diarrhea cases [8-10], in 50\%$70 \%$ of antibiotic-associated colitis cases, and in more than $90 \%$ of antibiotic-associated pseudomembranous colitis cases [11-15]. C. difficile-associated colitis (CDAC) is responsible for the majority of disease symptoms associated with profuse diarrhea, such as fever, leukocytosis, abdominal pain, and dehydration $[5,16]$.

A recent meta-analysis demonstrated that in Asia, the proportion of CDI in patients with nosocomial diarrhea was $14.8 \%$ and the associated mortality was found to be $8.9 \%$ [6]. Risk factors for CDI include the type of antibiotic, use of a combination of antibiotics, hospitalization, older age, underlying medical conditions, gastrointestinal surgery, and nasogastric tubes $[17,18]$. Antibiotics such as clindamycin, penicillin, fluoroquinolone, and second- and third-generation cephalosporin are typically associated with CDI, but the disease can occur with almost any anti-bacterial agent, including vancomycin and metronidazole, which are commonly used for treatment $[15,19,20]$.

Spontaneous reporting systems (SRSs) such as the Japanese Adverse Drug Event Report (JADER) database have been used in pharmacovigilance assessments. Based on an SRS database, data mining algorithms have been developed to identify drug-associated adverse events (AEs) as signals using reporting odds ratio (ROR) by employing a stepwise method. We previously reported that the concomitant use of drugs might increase the risk of AEs using these approaches [21,22].

Elderly individuals frequently have several chronic health conditions and are administered multiple medications, which is referred to as the number of administered drugs [21,23]. The number of administered anti-infectives can lead to drug interactions and may be an important risk factor for CDAC. It has been reported that antibiotic-associated diarrhea incidence was significantly higher in the combined drug-use group than in the monotherapy group [24]. To the best of our knowledge, the association between the number of concomitantly used antibiotics and CDAC remains unclear.

The clinical suspicion of CDI is the presentation of diarrhea after the administration of antibiotics shortly after the beginning of treatment and up to 12 weeks after treatment initiation [25-27]. Time-to-onset profiles of CDAC derived from the SRS databases have been rarely reported.

The aim of this study was to evaluate the antiinfective-related CDAC profiles by analyzing data from the SRS databases. We assessed the possible relationship among the number of concomitantly used antibiotics, reporting year, sex, and age at CDAC onset using adjusted RORs and a multiple-logistic regression technique. Furthermore, we obtained novel information on the time-to-onset profiles of CDAC relative to the anti-infective therapy initiation.

\section{Methods}

All data from the JADER database were fully anonymized by the regulatory authority of Japan; that is, the Pharmaceuticals and Medical Devices Agency (PMDA). The JADER database is publicly available and can be downloaded from the website of PMDA (www.pmda.go.jp). We assessed the JADER database between April 2004 and June 2018. The JADER database consists of four tables: 1) DEMO (patients' demographic information such as sex, age, and weight); 2) DRUG (drug name, and causality); 3) REAC (AEs and outcome); and, 4) HIST (medical history and primary illness). We integrated a relational database based on four data tables using FileMaker Pro 14 software (FileMaker, Santa Clara, CA, USA). In the DRUG table, the causality of each drug was assigned a code according to its association with AEs, such as "suspected drug," "concomitant drug," and "interacting drug." All cases of "suspected drug," "concomitant drug," or "interacting drug" were used in the analyses.

The AE definitions in the JADER database were provided by the Medical Dictionary for Regulatory Activities ver. 19.0/Japanese (MedDRA/J, www. pmrj.jp/jmo/php/indexj.php). We extracted reports of anti-infective-related CDAC using the following preferred terms (PTs): "Clostridium colitis" (PT code: 10058305), "Clostridium difficile colitis" (PT code: 10009657), "gastroenteritis clostridial" (PT code: 10017898), and "pseudomembranous colitis" (PT code: 10037128). According to the Anatomical Therapeutic Chemical (ATC) Classification System described by the World Health Organization Collaborating Centre for Drug Statistics Methodology (www.whocc.no/atc_ddd_index/), we verified anti-infectives and subsequently linked to the corresponding ATC classification codes. One hundred and thirty-six anti-infectives were selected and categorized into 32 ATC-drug classes (Table 1).

We calculated the crude RORs by comparing one of the index groups with the reference group. Each ROR was calculated from a two-by-two contingency table; it is the ratio of odds of reporting AEs versus all other events associated with the drug of interest compared with the reporting odds for all other drugs present in the database. The RORs are expressed as point estimates with 95\% confidence intervals (CIs). General qualitative judgments were used for signal detection, which depended on signal indices 
exceeding a predefined threshold. The ROR estimates of $<1$ indicate no potential exposure-event associations and estimates of $>1$ indicate potential exposure-event safety signals. A signal of the drug-event combination was detected when the lower limit of the $95 \%$ CI of the ROR exceeded 1 . Furthermore, $\geq 2$ cases were required to define the signal [28].

Table 1. Suspected drugs classified by the Anatomical Therapeutic Chemical classification system and the Defined Daily Dose (ATC/DDD)

Classification (ATC code) of anti-infectives Tetracyclines (J01AA)

Amphenicols (J01BA)

Penicillins with extended spectrum (J01CA)

Beta-lactamase sensitive penicillins (J01CE) Beta-lactamase resistant penicillins (J01CF) Beta-lactamase inhibitors (J01CG)

Combinations of penicillins, incl. beta-lactamase inhibitors (J01CR)

First-generation cephalosporins (J01DB)

Second-generation cephalosporins (J01DC)

Third-generation cephalosporins (J01DD)

Fourth-generation cephalosporins (J01DE) Monobactams (J01DF)

Carbapenems (J01DH)

Other cephalosporins and penems (J01DI) Trimethoprim and derivatives (J01EA)

Intermediate-acting sulfonamides (J01EC)

Long-acting sulfonamides (J01ED)

Combinations of sulfonamides and trimethoprim,

incl. derivatives (J01EE)

Macrolides (J01FA)

Lincosamides (J01FF)

Streptogramins (J01FG)

Streptomycins (J01GA)

Other aminoglycosides (J01GB)

Fluoroquinolones (J01MA

Other quinolones (J01MB)

Combinations of antibacterials (J01RA)

Glycopeptide antibacterials (J01XA)

Polymyxins (J01XB)

Steroid antibacterials $(\mathrm{J} 01 \mathrm{XC})$

Imidazole derivatives (J01XD)

Nitrofuran derivatives (J01XE)

Other antibacterials (J01XX)
Anti-infectives (Code No.)

demeclocycline (J01AA01), doxycycline (J01AA02), oxytetracycline (J01AA06), tetracycline (J01AA07), minocycline (J01AA08), tigecycline (J01AA12), combinations of tetracyclines (J01AA20)

chloramphenicol (J01BA01), thiamphenicol (J01BA02)

ampicillin (J01CA01), carbenicillin (J01CA03), amoxicillin (J01CA04), bacampicillin (J01CA06), piperacillin (J01CA12), talampicillin (J01CA15), sulbenicillin (J01CA16), aspoxicillin (J01CA19), ampicillin combinations (J01CA51)

benzylpenicillin (J01CE01), phenoxymethylpenicillin (J01CE02), pheneticillin (J01CE05)

cloxacillin (J01CF02)

sulbactam (J01CG01), tazobactam (J01CG02)

ampicillin and beta-lactamase inhibitor (J01CR01), sultamicillin (J01CR04), piperacillin and betalactamase inhibitor (J01CR05), combinations of penicillins (J01CR50)

cefalexin (J01DB01), cefaloridine (J01DB02), cefalotin (J01DB03), cefazolin (J01DB04), cefadroxil (J01DB05), cefatrizine (J01DB06), cefapirin (J01DB08), cefacetrile (J01DB10), cefroxadine (J01DB11)

cefoxitin (J01DC01), cefuroxime (J01DC02), cefaclor (J01DC04), cefotetan (J01DC05), cefotiam (J01DC07), cefmetazole (J01DC09), cefminox (J01DC12), cefbuperazone (J01DC13), flomoxef (J01DC14)

cefotaxime (J01DD01), ceftazidime (J01DD02), cefsulodin (J01DD03), ceftriaxone (J01DD04), cefmenoxime (J01DD05), latamoxef (J01DD06), ceftizoxime (J01DD07), cefixime (J01DD08), cefodizime (J01DD09), cefpiramide (J01DD11), cefoperazone (J01DD12), cefpodoxime (J01DD13), ceftibuten (J01DD14), cefdinir (J01DD15), cefditoren (J01DD16), cefcapene (J01DD17), cefoperazone and beta-lactamase inhibitor (J01DD62)

cefepime (J01DE01), cefpirome (J01DE02), cefozopran (J01DE03)

aztreonam (J01DF01), carumonam (J01DF02)

meropenem (J01DH02), doripenem (J01DH04), biapenem (J01DH05), imipenem and

cilastatin (J01DH51), panipenem and betamipron (J01DH55)

faropenem (J01DI03)

trimethoprim (J01EA01)

sulfamethoxazole (J01EC01), sulfadiazine (J01EC02)

sulfadimethoxine (J01ED01), sulfaphenazole (J01ED08)

sulfamethoxazole and trimethoprim (J01EE01)

erythromycin (J01FA01), spiramycin (J01FA02), midecamycin (J01FA03), roxithromycin (J01FA06), josamycin (J01FA07), clarithromycin (J01FA09), azithromycin (J01FA10), rokitamycin (J01FA12), telithromycin (J01FA15)

clindamycin (J01FF01), lincomycin (J01FF02)

quinupristin/dalfopristin (J01FG02)

streptomycin (J01GA01)

tobramycin (J01GB01), gentamicin (J01GB03), kanamycin (J01GB04), neomycin (J01GB05), amikacin (J01GB06), netilmicin (J01GB07), sisomicin (J01GB08), dibekacin (J01GB09), isepamicin (J01GB11), arbekacin (J01GB12), bekanamycin (J01GB13)

ofloxacin (J01MA01), ciprofloxacin (J01MA02), enoxacin (J01MA04), norfloxacin (J01MA06), lomefloxacin (J01MA07), fleroxacin (J01MA08), sparfloxacin (J01MA09),

levofloxacin (J01MA12), moxifloxacin (J01MA14), gatifloxacin (J01MA16), prulifloxacin (J01MA17), pazufloxacin (J01MA18), garenoxacin (J01MA19), sitafloxacin (J01MA21)

nalidixic acid (J01MB02), piromidic acid (J01MB03), pipemidic acid (J01MB04)

penicillins combinations with other antibacterials (J01RA01)

vancomycin (J01XA01), teicoplanin (J01XA02)

colistin (J01XB01), polymyxin B (J01XB02)

fusidic acid (J01XC01)

metronidazole (J01XD01), tinidazole (J01XD02)

nitrofurantoin (J01XE01)

fosfomycin (J01XX01), spectinomycin (J01XX04), mandelic acid (J01XX06),

linezolid (J01XX08), daptomycin (J01XX09), bacitracin (J01XX10) 
Table 2. Number of reports and crude reporting odds ratio of Clostridium difficile-associated colitis associated with anti-infectives

\begin{tabular}{|c|c|c|c|}
\hline Classification (ATC code) of anti-infectives & Total (n) & Case (n) & Crude ROR $(95 \% \mathrm{CI})$ \\
\hline Total & 534,688 & 1,222 & \\
\hline Tetracyclines (J01AA) & 1,763 & 15 & $3.78(2.27-6.30)^{\dagger}$ \\
\hline Amphenicols (J01BA) & 49 & 0 & - \\
\hline Penicillins with extended spectrum (J01CA) & 3,264 & 103 & $15.44(12.58-18.95)^{\dagger}$ \\
\hline Beta-lactamase sensitive penicillins (J01CE) & 93 & 2 & $9.61(2.36-39.05)^{\dagger}$ \\
\hline Beta-lactamase resistant penicillins ( $\mathrm{J} 01 \mathrm{CF}$ ) & 0 & 0 & $-{ }^{*}$ \\
\hline Beta-lactamase inhibitors (J01CG) & 0 & 0 & $-{ }^{*}$ \\
\hline Combinations of penicillins, incl. beta-lactamase inhibitors (J01CR) & 2,121 & 81 & $18.49(14.70-23.26)^{\dagger}$ \\
\hline First-generation cephalosporins (J01DB) & 1,484 & 49 & $15.49(11.58-20.71)^{\dagger}$ \\
\hline Second-generation cephalosporins (J01DC) & 1,902 & 79 & $20.16(15.97-25.43)^{\dagger}$ \\
\hline Third-generation cephalosporins (J01DD) & 7,551 & 313 & $25.03(21.97-28.52)^{\dagger}$ \\
\hline Fourth-generation cephalosporins (J01DE) & 1,318 & 61 & $22.25(17.10-28.95)^{\dagger}$ \\
\hline Monobactams (J01DF) & 21 & 2 & $46.03(10.71-197.82)^{\dagger}$ \\
\hline Carbapenems (J01DH) & 3,551 & 143 & $20.61(17.26-24.62)^{\dagger}$ \\
\hline Other cephalosporins and penems (J01DI) & 132 & 6 & $20.89(9.19-47.47)^{\dagger}$ \\
\hline Trimethoprim and derivatives (J01EA) & 0 & 0 & $-{ }^{*}$ \\
\hline Intermediate-acting sulfonamides (J01EC) & 36 & 0 & - \\
\hline Long-acting sulfonamides (J01ED) & 4 & 0 & $-{ }^{*}$ \\
\hline Combinations of sulfonamides and trimethoprim, incl. derivatives (J01EE) & 2,738 & 11 & $1.77(0.98-3.20)$ \\
\hline Macrolides (J01FA) & 5,283 & 146 & $13.96(11.71-16.62)^{\dagger}$ \\
\hline Lincosamides (J01FF) & 757 & 49 & $31.43(23.40-42.23)^{\dagger}$ \\
\hline Streptogramins (J01FG) & 2 & 0 & $-{ }^{*}$ \\
\hline Streptomycins (J01GA) & 153 & 0 & - \\
\hline Other aminoglycosides (J01GB) & 932 & 36 & $18.04(12.87-25.30)^{\dagger}$ \\
\hline Fluoroquinolones (J01MA) & 8,571 & 201 & $12.35(10.60-14.39)^{\dagger}$ \\
\hline Other quinolones (J01MB) & 24 & 0 & - \\
\hline Combinations of antibacterials (J01RA) & 638 & 8 & $5.57(2.77-11.22)^{\dagger}$ \\
\hline Glycopeptide antibacterials (J01XA) & 2,752 & 30 & $4.91(3.41-7.06)^{\dagger}$ \\
\hline Polymyxins (J01XB) & 137 & 1 & $-{ }^{*}$ \\
\hline Steroid antibacterials (J01XC) & 2 & 0 & - \\
\hline Imidazole derivatives (J01XD) & 667 & 17 & $11.56(7.12-18.78)^{\dagger}$ \\
\hline Nitrofuran derivatives (J01XE) & 0 & 0 & - \\
\hline Other antibacterials (J01XX) & 2,119 & 40 & $8.65(6.29-11.89)^{\dagger}$ \\
\hline
\end{tabular}

*Number of cases $<2$.

†Lower limit of $95 \%$ CI $>1$.

The use of RORs allowed adjustments by multiple-logistic regression analysis and offered the advantage of controlling covariates [29,30]. To calculate adjusted RORs, only reports with complete information of reporting year, age, and the number of administered anti-infectives were extracted. To construct the multiple-logistic regression model, reporting year, stratified age groups, and the number of administered anti-infectives were coded. The following multiple-logistic regression model was used in the analysis:

$$
\log (\text { odds })=\beta_{0}+\beta_{1} Y+\beta_{2} A+\beta_{3} N+\beta_{4} A \times N
$$

where, $Y$ is the reporting year, $A$ is the age-stratified group ( $<70$ years and $\geq 70$ years), and $\mathrm{N}$ is the number of administered anti-infectives. We evaluated the effects of explanatory variables using a stepwise method $[21,31]$ at a significance level of 0.05 (forward and backward). The contribution of selected variables in the final model was evaluated. The adjusted RORs were calculated using the multiple-logistic regression model. A likelihood ratio test was used to evaluate the effects of explanatory variables.

Most developed world countries and the World Health Organization (WHO) have accepted the chronological age of 65 years as a definition of "elderly" or older person. The description of age was recorded in the data table of DEMO. The reports were stratified by age as follows: $\leq 19,20-29,30-39,40-49$, $50-59,60-69,70-79,80-89$, and $\geq 90$ years. For the calculation of the adjusted ROR, the reports were stratified by age as $<70$ and $\geq 70$ years, because 65 years was categorized into precise 10-year intervals in the JADER database. Neonate, baby, infant, child, young adult, and women in the first-, second-, and third-trimester of pregnancy were categorized into the $<70$-year-old group. We excluded elderly, adults, and unknown items because these descriptions could not be precisely categorized into the $<70$ and 
$\geq 70$-year-old groups. The number of administered anti-infectives was categorized as 1,2 , and $\geq 3$ drugs.

Time-to-onset duration was obtained from the CDAC onset date to the time of the first prescription date for each anti-infective. The median duration, interquartile range, and Weibull shape parameter (WSP) were used to evaluate the time-to-onset profile. The analysis period was 90 days after the first prescription date. The rate of $\mathrm{AE}$ occurrence after prescription is assumed to depend on a causal mechanism and often varies with time; in contrast, AEs not associated with the drug should occur at a constant background rate. The WSP test was used for the statistical analysis of time-to-onset data to describe a non-constant ratio of the incidence of AEs. The WSP was used to describe the varying incidence of AEs and to evaluate hazard functions for detecting AEs. The scale parameter a of Weibull distribution determines the scale of the distribution function. A larger scale value (a) stretches data distribution, whereas a smaller scale value shrinks data distribution. The shape parameter $\beta$ of Weibull distribution determines the shape of the distribution function. The WSP $\beta$ value indicates the hazard without a reference population; when $\beta$ is equal to 1 , the hazard is estimated to be constant over time. If $\beta$ is greater than 1 and the $95 \% \mathrm{CI}$ of $\beta$ excludes the value of 1 , the hazard is considered to increase over time $[29,32,33]$. Information using WSP could be of complementary value for the pharmacovigilance analysis using ROR. All data analyses were performed using JMP 12.0 (SAS Institute, Cary, NC, USA).

\section{Results}

The JADER database contains 534688 reports submitted between April 2004 and June 2018, and we identified 1222 anti-infective-related CDAC. The top five anti-infectives that cause CDAC-associated AEs were third-generation cephalosporins (ATC code: J01DD, 313 cases), fluoroquinolones (ATC code: J01MA, 201 cases), macrolides (ATC code: J01FA, 146 cases), carbapenems (ATC code: J01DH, 143 cases), and penicillins with extended spectrum (ATC code: J01CA, 103 cases) (Table 2). The drug groups for which the lower limits of $95 \%$ CI of RORs were $>1$ and RORs were $>10$ were as follows: penicillins with extended spectrum (ATC code: J01CA); combinations of penicillins, including beta-lactamase inhibitors (ATC code: J01CR); first-generation cephalosporins (ATC code: J01DB); second-generation cephalosporins (ATC code: J01DC); third-generation cephalosporins (ATC code: J01DD); fourth-generation cephalosporins (ATC code: J01DE); monobactams (ATC code: J01DF); carbapenems (ATC code: J01DH); other cephalosporins and penems (ATC code: J01DI); macrolides (ATC code: J01FA); lincosamides (ATC code: J01FF); other aminoglycosides (ATC code: J01GB); fluoroquinolones (ATC code: J01MA); and imidazole derivatives (ATC code: J01XD) (Table 2).

For the time-to-onset analysis, we extracted combinations that had complete information on the date of treatment initiation and the date of $\mathrm{AE}$ onset. We evaluated 16 anti-infective categories. Figure 1 shows a histogram of the number of CDAC onsets in relation to the number of days after anti-infective treatment initiation (from days 0 to 90). The median period (interquartile range) until CDAC onset caused by anti-infectives was $7.0(3.0-16.0)$ days for all anti-infectives (Table 3). The lower limit of the $95 \%$ CI of WSP $\beta$ was $>1$ for the following drug groups: combinations of penicillins, including beta-lactamase inhibitors (ATC code: J01CR); third-generation cephalosporins (ATC code: J01DD); fourth-generation cephalosporins (ATC code: J01DE); and other cephalosporins, and penems (ATC code: J01DI).

The adjusted RORs and 95\% CIs are summarized in Table 4. The results of the model indicated significant contributions of the reporting year $(p=$ 0.0013 ) and the number of administered antiinfectives $(1,2$, and $\geq 3$ drugs, $p<0.0001)$. The adjusted RORs of the anti-infectives 1,2 , and $\geq 3$ drugs were 8.88 (7.05-11.18), 9.77 (6.89-13.86), and 18.39 (11.85$28.54)$, respectively. The adjusted ROR of interaction terms of $\geq 70$ years $\times 1$ drug was 21.81 (14.56-32.68).

\section{Discussion}

CDAC, such as pseudomembranous colitis, is a serious colonic disease, which can occur when antibiotics or other agents disrupt the normal colonic flora [34]. Almost all classes of anti-infectives increased CDI risk; certain classes including clindamycin, fluoroquinolones, and cephalosporins might have a higher CDI risk than tetracycline [19,3436]. In this study, AE signals indicating an association with CDAC were detected in many categories of anti-infectives such as penicillins with extended spectrum (ATC code: J01CA), first to fourthgeneration cephalosporins (ATC code: J01DB, J01DC, J01DD, and J01DE), lincosamides (ATC code: J01FF), and fluoroquinolones (ATC code: J01MA), which agrees with previous findings (Table 2) [36].

CDI can occur after a few days of antibiotic therapy or up to 3 months after use [25-27,37]. The median for anti-infective-related CDAC onset was 7 days post-initiation. Furthermore, $47.2 \%$ of antiinfective-related CDAC occurred within 7 days of treatment. We did not detect significant differences in time-to-onset profiles among 16 ATC-drug classes of anti-infectives. 
1) Total

Median (quartiles, day): 7.0 (3.0-16.0) $\beta$ (95\% Cl): 1.07 (1.01-1.14)

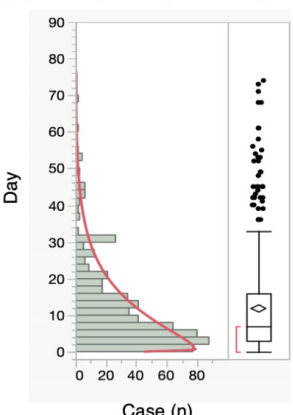

Case (n)

5) Second-generation cephalosporins (J01DC)

Median (quartiles, day): 8.5 (3.0-15.3) $\beta(95 \% \mathrm{Cl}): 1.06(0.82-1.33)$

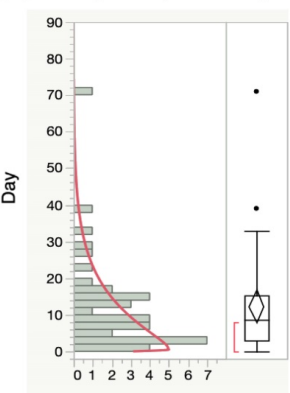

Case (n)

9) Other cephalosporins and penems (J01DI)

Median (quartiles, day): 8.0 (1.0-12.0) $\beta$ (95\% Cl): 4.81 (1.71-10.35)

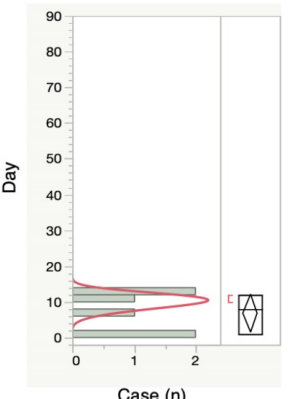

13) Fluoroquinolones (J01MA)

Median (quartiles, day): 7.0 (3.0-14.0) $\beta(95 \% \mathrm{Cl}): 1.03(0.88-1.21)$

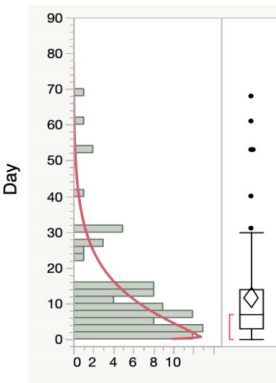

Case (n)
2) Penicillins with extended spectrum (J01CA)

Median (quartiles, day): 11.0 (5.0-18.5) $\beta$ (95\% Cl): 1.05 (0.77-1.38)

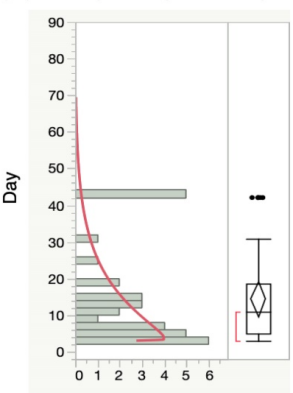

Case (n)

6) Third-generation cephalosporins (J01DD)

Median (quartiles, day): 6.0 (3.0-13.0) $\beta$ (95\% Cl): 1.23 (1.07-1.41)

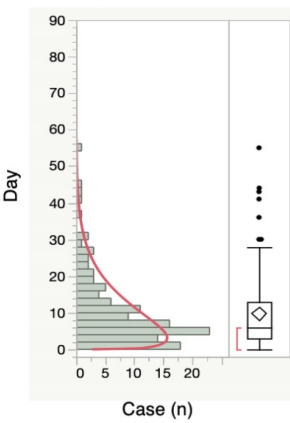

10) Macrolides (J01FA)

Median (quartiles, day): 5.0 (3.0-13.0) $\beta$ (95\% Cl): $0.95(0.77-1.15)$

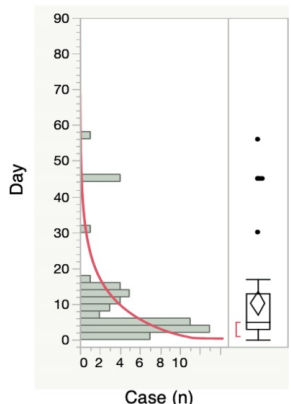

14) Combinations of antibacterials (J01RA)

Median (quartiles, day): 4.5 (2.5-10.5) $\beta(95 \% \mathrm{Cl}): 1.44$ (0.63-2.63)

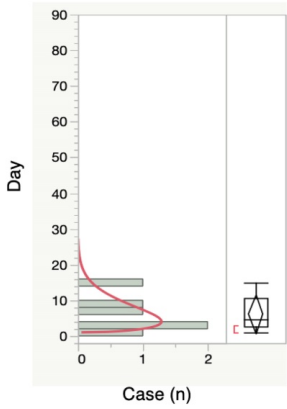

3) Combinations of penicillins, 4) First-generation cephalosporins incl. beta-lactamase inhibitors (J01DB)

$$
\text { (J01CR) }
$$

Median (quartiles, day): 13.0 (4.0-21.0) Median (quartiles, day): 6.0 (3.5-15.0) $\beta(95 \% \mathrm{Cl}): 1.31(1.01-1.66)$

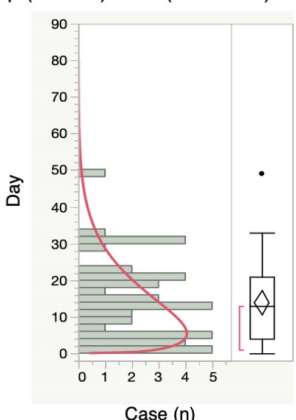

$\beta(95 \% \mathrm{Cl}): 1.23(0.85-1.69)$

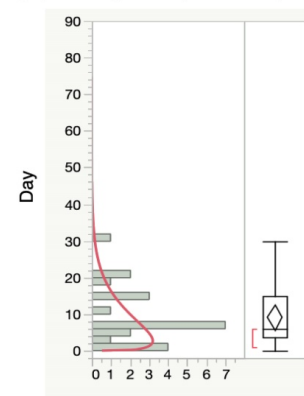

Case (n)

\section{7) Fourth-generation cephalosporins (J01DE)}

Median (quartiles, day): $7.0(4.0-11.8)$ $\beta(95 \% \mathrm{Cl}): 1.49(1.11-1.94)$

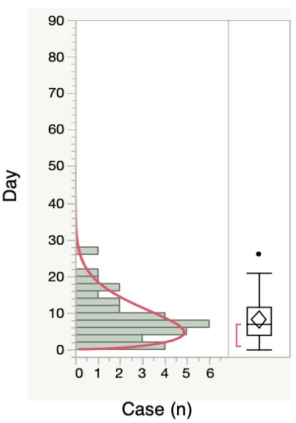

11) Lincosamides (J01FF)

Median (quartiles, day): $4.0(2.0-24.8)$ $\beta(95 \% \mathrm{Cl}): 0.73(0.42-1.14)$

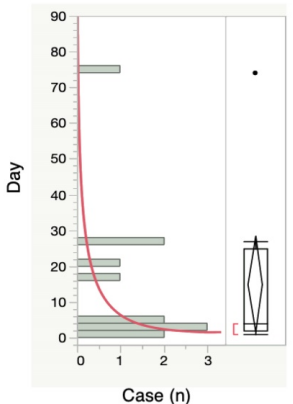

15) Glycopeptide antibacterials (J01XA)

Median (quartiles, day): $16.0(1.0-23.0)$ $\beta(95 \% \mathrm{Cl}): 1.11(0.61-1.81)$

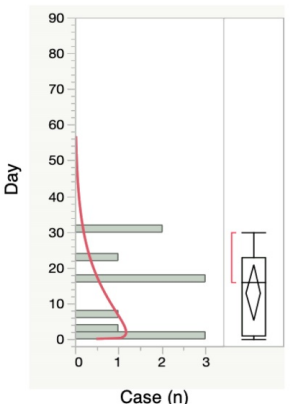

\section{8) Carbapenems (J01DH)}

Median (quartiles, day): 6.5 (2.8-18.3) $\beta(95 \% \mathrm{Cl}): 1.01$ (0.83-1.21)

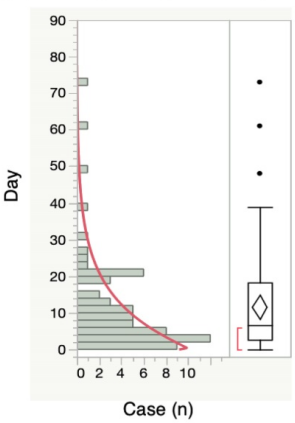

12) Other aminoglycosides (J01GB)

Median (quartiles, day): 28.0 (8.5-30.0) $\beta$ (95\% Cl): 1.60 (1.04-2.29)

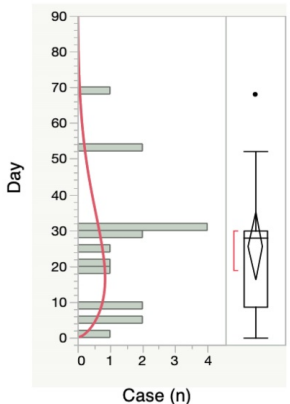

Median (quartiles, day): $1.5(0.0-7.5)$ $\beta(95 \% \mathrm{Cl}): 2.01(0.64-4.37)$

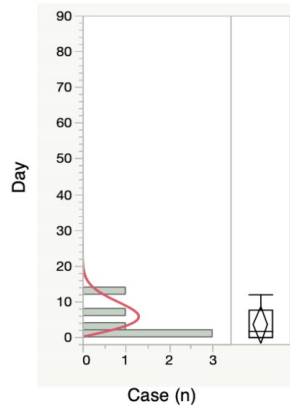

16) Imidazole derivatives (J01XD) 


\section{7) Other antibacterials (J01XX)}

\section{Median (quartiles, day): 17.0 (4.0-30.0)}

$\beta$ (95\% Cl): 1.38 (0.89-1.99)

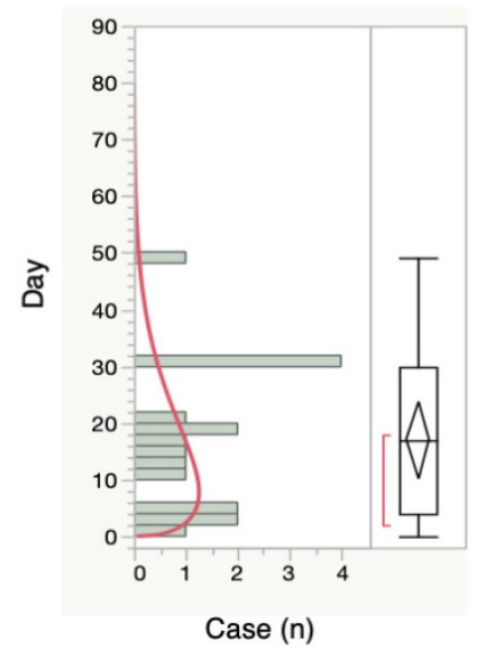

Figure 1. Time-to-onset profiles of anti-infective-related Clostridium difficile-associated colitis in the Japanese Adverse Event Report (JADER) database. The records with complete adverse event occurrence and prescription star date were used for the time-to-onset analysis.

Table 3. The medians and Weibull parameter of anti-infectives

\begin{tabular}{lrrrr}
\hline Classification (ATC code) & Case Median (day) & \multicolumn{1}{l}{ Scale parameter } & \multicolumn{2}{l}{ Shape parameter } \\
\cline { 4 - 6 } & (n) $(25 \%-75 \%)$ & \multicolumn{1}{l}{$\alpha(95 \% \mathrm{CI})$} & \multicolumn{1}{l}{$\beta(95 \% \mathrm{CI})$} \\
\hline Total & 615 & $7.0(3.0-16.0)$ & $13.17(12.14-14.27)$ & $1.07(1.01-1.14)$ \\
Penicillins with extended spectrum (J01CA) & 33 & $11.0(5.0-18.5)$ & $14.36(9.57-21.08)$ & $1.05(0.77-1.38)$ \\
Combinations of penicillins, incl. beta-lactamase inhibitors (J01CR) & 44 & $13.0(4.0-21.0)$ & $15.94(12.36-20.32)$ & $1.31(1.01-1.66)$ \\
First-generation cephalosporins (J01DB) & 22 & $6.0(3.5-15.0)$ & $10.25(6.90-14.89)$ & $1.23(0.85-1.69)$ \\
Second-generation cephalosporins (J01DC) & 38 & $8.5(3.0-15.3)$ & $13.03(9.31-17.98)$ & $1.06(0.82-1.33)$ \\
Third-generation cephalosporins (J01DD) & 127 & $6.0(3.0-13.0)$ & $12.07(10.26-14.15)$ & $1.23(1.07-1.41)$ \\
Fourth-generation cephalosporins (J01DE) & 32 & $7.0(4.0-11.8)$ & $9.76(7.46-12.60)$ & $1.49(1.11-1.94)$ \\
Carbapenems (J01DH) & 66 & $6.5(2.8-18.3)$ & $12.65(9.64-16.45)$ & $1.01(0.83-1.21)$ \\
Other cephalosporins and penems (J01DI) & 6 & $8.0(1.0-12.0)$ & $9.89(7.28-13.32)$ & $4.81(1.71-10.35)$ \\
Macrolides (J01FA) & 56 & $5.0(3.0-13.0)$ & $10.39(7.63-13.98)$ & $0.95(0.77-1.15)$ \\
Lincosamides (J01FF) & 12 & $4.0(2.0-24.8)$ & $13.81(4.95-35.95)$ & $0.73(0.42-1.14)$ \\
Other aminoglycosides (J01GB) & 19 & $24.0(5.0-30.0)$ & $26.28(17.24-39.09)$ & $1.27(0.84-1.79)$ \\
Fluoroquinolones (J01MA) & 99 & $7.0(3.0-14.0)$ & $12.64(10.22-15.53)$ & $1.03(0.88-1.20)$ \\
Combinations of antibacterials (J01RA) & 6 & $4.5(2.5-10.5)$ & $6.87(3.02-14.97)$ & $1.44(0.63-2.63)$ \\
Glycopeptide antibacterials (J01XA) & 11 & $16.0(1.0-23.0)$ & $14.90(7.48-28.36)$ & $1.11(0.61-1.81)$ \\
Imidazole derivatives (J01XD) & 6 & $1.5(0.0-7.5)$ & $7.95(3.31-18.90)$ & $2.01(0.64-4.37)$ \\
Other antibacterials (J01XX) & 17 & $17.0(4.0-30.0)$ & $19.84(13.11-29.29)$ & $1.38(0.89-1.99)$ \\
\hline
\end{tabular}

Table 4. Adjusted reporting odds ratio of Clostridium difficile-associated colitis

\begin{tabular}{lrrr}
\hline & Estimated beta & Adjusted $\operatorname{ROR}^{*}\left(95 \% \mathrm{Cl}^{\dagger}\right)$ & P-value $^{\ddagger}$ \\
\hline Total & & & \\
$\begin{array}{l}\text { Reporting year } \\
\text { Age }\end{array}$ & -0.041 & $0.56(0.55-0.57)$ & $0.0013^{\S}$ \\
$\quad$ & & & \\
$\quad \geq 70$ years & 0.129 & $1.14(0.86-1.50)$ & 0.3640 \\
Anti-infectives & & & \\
$\quad 1$ drug & 2.184 & $8.88(7.05-11.18)$ & $<0.0001^{\S}$ \\
$\quad 2$ drugs & 2.280 & $9.77(6.89-13.86)$ & $<0.0001^{\S}$ \\
$\quad \geq 3$ drugs & 2.912 & $18.39(11.85-28.54)$ & $<0.0001^{\S}$ \\
Interaction terms & & & \\
$\quad \geq 70$ years $\times 1$ drug & 0.770 & $21.81(14.56-32.68)$ & $0.0005^{\S}$ \\
\hline
\end{tabular}

*Reporting Odds Ratio; †Confidence interval;

$\ddagger$ Probability $>$ Chi-square; $\delta P<0.05$. 
The adjusted risk associated with CDI was similar when patients received either a single class of anti-infectives (incidence rate ratio $=2.49,95 \% \mathrm{CI}$ 1.59-3.92) or multiple classes of anti-infectives (incidence rate ratio $=2.09,95 \%$ CI 1.23-3.55) [38]. Contrarily, in a retrospective cohort study, compared with that for patients who received only 1 antibiotic, the adjusted hazard ratios (HRs) of CDI for those who received 2, 3 or 4 , or 5 or more antibiotics were 2.5 (95\% CI 1.6-4.0), 3.3 (95\% CI 2.2- 5.2), and 9.6 (95\% CI 6.1-15.1), respectively [39]. Anti-infective-related diarrhea has been reported to be at higher risk in combination therapy than in monotherapy [24]. Therefore, we evaluated the risk of CDAC in the real-world clinical setting. The adjusted ROR of anti-infective-related CDAC increased with the number of anti-infectives (Table 4). It is thought that the combination of multiple anti-infectives broadens the antibacterial spectrum and disturbs the intestinal bacterial flora. Avoiding unnecessary antibiotic exposure is often adopted as one of standard preventive strategies for CDI [38].

CDAC is highly common in adults, especially in elderly patients [40]. The frequently reported risk factor for recurrent CDI is older age [41-44]. We applied the multiple-logistic regression analysis to validate the results. The interaction terms of $\geq 70$ years $\times 1$ drug for CDAC was significant in the JADER database.

SRS is a passive reporting system and does not contain detailed patient background to appropriately evaluate an event. SRS is subject to a lack of a comparison group, over-reporting, under-reporting, missing data, and presence of confounders. For example, since the JADER database is based on spontaneous reports, healthcare "professionals" may not usually report CDI to this database. Therefore, the number of cases of CDI might be underestimated.

Proton pump inhibitors and $\mathrm{H}_{2}$-blockers have also been identified to increase the risk of acquiring CDI, although the causality and precise mechanism remains unclear. [41,45-48] The effects of these concomitant medications have not been evaluated. The risk of CDI is associated with increasing total dose and days of antibiotic exposure [39,49]. It is reported that the incidence rates of CDI following 1-3, 4-6, and 7-11 days of cephalosporin exposure were $1.60,2.27$, and 2.10 times higher than those in no prior receipt, respectively [38]. Other risk factors for CDI include the use of a co-administered proton pomp inhibitor and the length of hospital stay $[18,24]$. In this study, we did not evaluate the effect of these factors. Considering the causality restraints of the present analysis, further robust epidemiological studies are recommended.
The association between the number of administered anti-infectives and the onset duration of CDI is interesting. However, when two or more anti-infectives are administered simultaneously, it is difficult to accurately determine the start date of drug administration because the start date cannot be uniquely determined in the JADER database. Therefore, the JADER database is not suitable for analyzing relatively short-term AEs such as CDAC. The median period (interquartile range) until CDAC onset caused by anti-infectives of 1,2 , and $\geq 3$ drugs were apparently $3.0(1.0-6.0)(\mathrm{n}=191), 5.0(3.0-8.8)(\mathrm{n}$ $=48)$, and $10.0(2.0-21.0)(\mathrm{n}=23)$ days. We think that this result may not accurately reflect the association between the number of administered anti-infectives and the onset of CDI. Therefore, the interpretation of this result requires great care.

To assist terminology searches, MedDRA is used in post-marketing surveillance. Standardised MedDRA Queries (SMQs) were built by the Maintenance and Support Services Organization. SMQs are groupings of PTs according to the level that relates to a defined medical condition, and the included terms may relate to signs, symptoms, diagnoses, syndromes, physical findings, and laboratory and other physiological test data [50]. The grouping of SMQs allows for useful data retrieval and the presentation of relevant individual case safety reports. In the research of CDI in the FDA adverse event reporting system (FAERS), PTs based on pseudomembranous colitis (SMQs) such as "Clostridial infection," "Clostridial sepsis," "Clostridium bacteraemia," "Clostridium colitis," "Clostridium difficile colitis," "Clostridium difficile infection," "Clostridium test positive," "Gastroenteritis clostridial," "Pseudomembranous colitis" and "Clostridium difficile sepsis," which are the lowest level terms, were used to identify CDI cases [36]. The choice of term should be made in accordance with the purpose of the study, and thus, our choice of term was focused on CDAC. Therefore, in our study, PTs of "Clostridial infection," "Clostridial sepsis," "Clostridium bacteremia," Clostridium difficile infection," "Clostridium test positive," and "Clostridium difficile sepsis" were excluded. Generally, with a narrow selection of PTs, the identification of cases is highly likely to represent the condition of interest, and if PTs are selected broadly, the identification of cases might contain all possible cases, including some that may prove to be of little or no interest on closer inspection. The "narrow" scope yields "specificity," while the "broad" search yields "sensitivity." [50] We consider that these data suggest the association of certain drugs with CDAC. However, the calculated RORs might vary 
significantly depending on the selection of PTs, and further validation of these associations is needed.

The diagnosis of CDI is necessary to detect the toxin. However, it is difficult to confirm the diagnosis of CDI directly from the JADER database. We selected PTs for the identification of CDAC based on MedDRA. According to the Introductory Guide MedDRA Version 19.0, each PT is a distinct descriptor (a single medical concept) for a symptom; sign; disease; diagnosis; therapeutic indication; investigation; surgical or medical procedure; or a medical, social, or family history characteristic [51]. The JADER database is derived from spontaneous volunteer reporting. The contributors only report AEs according to ICH E2B, the international safety reporting guidelines, and rely on the definitions provided by MedDRA. It might be difficult to confirm the criteria used to define CDAC events by volunteers at the time of reporting. However, the data in the JADER database have been reported by healthcare "professionals." Therefore, we believe the results are worthy of evaluation.

\section{Conclusions}

To the best of our knowledge, this is the first study to evaluate anti-infective-related CDAC associated with the number of administered antiinfectives and aging using an SRS analysis strategy. Based on the ROR, we demonstrated the potential CDAC risk related to numerous anti-infectives including penicillins with extended spectrum, first- to fourth-generation cephalosporins, lincosamides, and fluoroquinolones. Adjusted RORs demonstrated that the number of administered anti-infectives and aging might be more closely associated with an increasing risk of CDAC. The median period until anti-infectiverelated CDAC onset was 7 days after therapy initiation. We believe that the data presented in this study will provide guidance for healthcare professionals to improve the care of elderly patients receiving different medications concomitantly.

\section{Acknowledgments}

\section{Ethical approval}

Ethical approval was not sought for this study because the study was an observational study without any research subjects. All results were obtained from data openly available online from the PMDA website. All data from the JADER database were fully anonymized by the regulatory authority before we accessed them.

\section{Funding}

The author(s) disclosed receipt of the following financial support for the research, authorship, and/or publication of this article. This research was partially supported by the Japan Society for the Promotion of Science KAKENHI grant number, 17K08452. The funders had no role in the study design, data collection and analysis, decision to publish, or preparation of the article.

\section{Grant number}

This research was partially supported by JSPS KAKENHI Grant Number, 17K08452.

\section{Competing Interests}

The authors have declared that no competing interest exists.

\section{References}

1. Hull MW, Beck PL. Clostridium difficile-associated colitis. Can Fam Physician. 2004; 50: 1536-1540, 1543-1545.

2. Leffler DA, Lamont JT. Clostridium difficile infection. N Engl J Med. 2015; 372: 1539-1548.

3. Henriksson G, Bredberg J, Wullt M, et al. Humoral response to Clostridium difficile in inflammatory bowel disease, including correlation with immunomodulatory treatment. JGH Open. 2019; 3: 154-158.

4. Abt MC, McKenney PT, Pamer EG. Clostridium difficile colitis: pathogenesis and host defence. Nat Rev Microbiol. 2016; 14: 609-620.

5. Masciullo V, Mainenti S, Lorusso D, et al. Lethal Clostridium difficile colitis associated with paclitaxel and carboplatin chemotherapy in ovarian carcinoma: case report and review of the literature. Obstet Gynecol Int. 2010; 2010: 749789 .

6. Borren NZ, Ghadermarzi S, Hutfless S, et al. The emergence of Clostridium difficile infection in Asia: A systematic review and meta-analysis of incidence and impact. PLoS One. 2017; 12: e0176797.

7. Gerding DN. Disease associated with Clostridium difficile infection. Ann Intern Med. 1989; 110: 255-257.

8. Sun YX, Zhao YT, Teng LL, et al. Clostridium difficile infection associated with antituberculous agents in a patient with tuberculous pericarditis. Intern Med. 2013; 52: 1495-1497.

9. Bouza E, Burillo A, Muñoz P. Antimicrobial Therapy of Clostridium difficile-associated diarrhea. Med Clin North Am. 2006; 90: 1141-1163.

10. Bartlett JG. Antibiotic-associated diarrhea. Clin Infect Dis. 1992; 15: 573-581.

11. Bartlett JG, Taylor NS, Chang T, et al. Clinical and laboratory observations in Clostridium difficile colitis. Am J Clin Nutr. 1980; 33 (11 Suppl): 2521-2526.

12. Bartlett JG. Clostridium difficile: clinical considerations. Rev Infect Dis. 1990; 12(Suppl 2): S243-251.

13. Bartlett JG. Clinical practice. Antibiotic-associated diarrhea. N Engl J Med. 2002; 346: 334-339.

14. George WL, Rolfe RD, Finegold SM. Clostridium difficile and its cytotoxin in feces of patients with antimicrobial agent-associated diarrhea and miscellaneous conditions. J Clin Microbiol. 1982; 15: 1049-1053.

15. Kelly CP, Pothoulakis C, LaMont JT. Clostridium difficile colitis. N Engl J Med. 1994; 330: 257-262.

16. Ash L, Baker ME, O'Malley CM Jr, et al. Colonic abnormalities on CT in adult hospitalized patients with Clostridium difficile colitis: prevalence and significance of findings. AJR Am J Roentgenol. 2006; 186: 1393-1400.

17. Lv X, Zhang J, Jiang $\mathrm{M}$, et al. Clostridium difficile-associated diarrhea following the therapy with antibiotic and proton pump inhibitors in a 77-year-old man with several comorbidities: A case report. Medicine (Baltimore). 2019; 98: e15004.

18. Ruiter-Ligeti J, Vincent S, Czuzoj-Shulman N, et al. Risk factors, incidence, and morbidity associated with obstetric Clostridium difficile infection. Obstet Gynecol. 2018; 131: 387-391.

19. Farooq PD, Urrunaga NH, Tang DM, et al. Pseudomembranous colitis. Dis Mon. 2015; 61: 181-206.

20. Mylonakis E, Ryan ET, Calderwood SB. Clostridium difficile-Associated diarrhea: A review. Arch Intern Med. 2001; 161: 525-533.

21. Abe J, Umetsu R, Uranishi H, et al. Analysis of polypharmacy effects in older patients using Japanese Adverse Drug Event Report database. PLoS One. 2017; 12: e0190102.

22. Hatahira H, Hasegawa S, Sasaoka S, et al. Analysis of fall-related adverse events among older adults using the Japanese Adverse Drug Event Report (JADER) database. J Pharm Health Care Sci. 2018; 4: 32.

23. Charlesworth CI, Smit E, Lee DSH, et al. Polypharmacy among adults aged 65 years and older in the United States: 1988-2010. J Gerontol A Biol Sci Med Sci. 2015; 70: 989-995. 
24. Ma H, Zhang L, Zhang $\mathrm{Y}$, et al. Combined administration of antibiotics increases the incidence of antibiotic-associated diarrhea in critically ill patients. Infect Drug Resist. 2019; 12: 1047-1054.

25. Bartlett JG, Gerding DN. Clinical recognition and diagnosis of Clostridium difficile infection. Clin Infect Dis. 2008; 46 Suppl 1: S12-S18.

26. Surawicz CM, Brandt LJ, Binion DG, et al. Guidelines for diagnosis, treatment, and prevention of Clostridium difficile infections. Am J Gastroenterol. 2013; 108: 478-498.

27. Hensgens MP, Goorhuis A, Dekkers OM, et al. Time interval of increased risk for Clostridium difficile infection after exposure to antibiotics. J Antimicrob Chemother. 2012; 67: 742-748.

28. Bate A, Evans SJW. Quantitative signal detection using spontaneous ADR reporting. Pharmacoepidemiol Drug Saf. 2009; 18: 427-436.

29. Tanaka M, Hasegawa S, Nakao S, et al. Analysis of drug-induced hearing loss by using a spontaneous reporting system database. PLoS One. 2019; 14: e0217951.

30. Shimada K, Hasegawa S, Nakao $S$, et al. Adverse event profiles of ifosfamide-induced encephalopathy analyzed using the Food and Drug Administration Adverse Event Reporting System and the Japanese Adverse Drug Event Report databases. Cancer Chemother Pharmacol. 2019; 84: 10971105.

31. Takeyama M, Sai K, Imatoh T, et al. Influence of Japanese regulatory action on denosumab-related hypocalcemia using Japanese Adverse Drug Event Report database. Biol Pharm Bull. 2017; 40: 1447-1453.

32. Hatahira H, Abe J, Hane $Y$, et al. Drug-induced gingival hyperplasia: a retrospective study using spontaneous reporting system databases. J Pharm Heal care Sci. 2017; 3: 19.

33. Sauzet O, Carvajal A, Escudero A, et al. Illustration of the weibull shape parameter signal detection tool using electronic healthcare record data. Drug Saf. 2013; 36: 995-1006.

34. Surawicz CM, McFarland LV. Pseudomembranous colitis: causes and cures. Digestion. 1999; 60: 91-100.

35. Delaney JAC, Dial S, Barkun A, et al. Antimicrobial drugs and community-acquired Clostridium difficile-associated disease, UK. Emerg Infect Dis. 2007; 13: 761-763.

36. Teng C, Reveles KR, Obodozie-Ofoegbu OO, et al. Clostridium difficile infection risk with important antibiotic classes: an analysis of the FDA Adverse Event Reporting System. Int J Med Sci. 2019; 16: 630-635.

37. Fekety R. Guidelines for the diagnosis and management of Clostridium difficile-associated diarrhea and colitis. American College of Gastroenterology, Practice Parameters Committee. Am J Gastroenterol. 1997; 92: 739-750.

38. Brown KA, Fisman DN, Moineddin R, et al. The magnitude and duration of Clostridium difficile infection risk associated with antibiotic therapy: A hospital cohort study. PLoS One. 2014; 9: e105454.

39. Stevens V, Dumyati G, Fine LS, et al. Cumulative antibiotic exposures over time and the risk of Clostridium difficile infection. Clin Infect Dis. 2011; 53: 4248 .

40. Gebhard RL, Gerding DN, Olson MM, et al. Clinical and endoscopic findings in patients early in the course of Clostridium difficile-associated pseudomembranous colitis. Am J Med. 1985; 78: 45-48.

41. Johnson S. Recurrent Clostridium difficile infection: a review of risk factors, treatments, and outcomes. J Infect. 2009; 58: 403-410.

42. Deshpande A, Pasupuleti V, Thota P, et al. Risk factors for recurrent Clostridium difficile infection: A systematic review and meta-analysis. Infect Control Hosp Epidemiol. 2015; 36: 452-460.

43. Abou Chakra CN, Pepin J, Sirard S, et al. Risk factors for recurrence, complications and mortality in Clostridium difficile infection: A systematic review. PLoS One. 2014; 9: e98400.

44. Garey KW, Sethi S, Yadav Y, et al. Meta-analysis to assess risk factors for recurrent Clostridium difficile infection. J Hosp Infect. 2008; 70: 298-304.

45. Loo VG, Bourgault AM, Poirier L, et al. Host and pathogen factors for Clostridium difficile infection and colonization. N Engl J Med. 2011; 365: 16931703.

46. Garey KW, Jiang ZD, Ghantoji S, et al. A common polymorphism in the interleukin-8 gene promoter is associated with an increased risk for recurrent Clostridium difficile infection. Clin Infect Dis. 2010; 51: 1406-1410.

47. Kelly CP, LaMont JT. Clostridium difficile-more difficult than ever. N Engl J Med. 2008; 359: 1932-1940.

48. Croft L, Ladd J, Doll M, et al. Inappropriate Antibiotic Use and Gastric Acid Suppression Preceding Clostridium difficile Infection. Infect Control Hosp Epidemiol. 2016; 37: 494-495.

49. Stevens $\mathrm{V}$, van Wijngaarden E. Ignoring the variability in timing of drug administrations attenuates hazard rations. Am J Epidemiol. 2011; 173: S272.

50. [Internet] MedDRA MSSO. Introductory Guide for Standardized MedDRA Queries (SMQs) Version 19.0. 2016. http://www.meddra.org/sites/ default/files/guidance/file/smq_intguide_19_0_english.pdf

51. [Internet] International Council for Harmonization, Introductory Guide MedDRA Version 19.0. https://www.meddra.org/sites/default/ files/guidance/file/intguide_19_0_english.pdf 